\title{
Anabases
}

ANABASES Traditions et réceptions de l'Antiquité

$12 \mid 2010$

Varia

\section{Berliner Konstellationen am Ende der Weimarer Republik. Eduard Norden und die Altertumswissenschaft in Jerusalem}

Hubert Cancik et Hildegard Cancik-Lindemaier

\section{OpenEdition}

Journals

Édition électronique

URL : http://journals.openedition.org/anabases/1089

DOI : 10.4000/anabases.1089

ISSN : 2256-9421

Éditeur

E.R.A.S.M.E.

Édition imprimée

Date de publication : 1 octobre 2010

Pagination : 69-91

ISSN : $1774-4296$

Référence électronique

Hubert Cancik und Hildegard Cancik-Lindemaier, « Berliner Konstellationen am Ende der Weimarer Republik. Eduard Norden und die Altertumswissenschaft in Jerusalem », Anabases [Online], 12 | 2010, Online erschienen am: 01 Oktober 2013, besucht am 21 Oktober 2019. URL : http:// journals.openedition.org/anabases/1089; DOI : 10.4000/anabases.1089 


\section{Berliner Konstellationen am Ende der Weimarer Republik. Eduard Norden und die Altertumswissenschaft in Jerusalem}

Hubert CANCiK

HiLDEgard CANCIK-LindeMAIER

\section{Eduard Norden als Rektor ${ }^{1}$}

\subsection{Universitärer Glanz}

Das Bild Eduard Nordens, das kürzlich im Institut für Klassische Philologie der Humboldt-Universität zu Berlin aufgehängt wurde (Abb. 1), zeigt den sechzigjährigen Gelehrten auf dem Höhepunkt seiner öffentlichen Wirksamkeit, in seinem Rektoratsjahr (15. Oktober 1927 bis 14. Oktober 1928²). In diesem Jahre, am

1 Für Auskünfte und mancherlei Hilfe danken wir unseren Berliner KollegInnen Bernhard Kytzler, Wolfgang Rösler, Ulrich Schmitzer, Isolde Stark. Peter Norden hat uns den persönlichen und wissenschaftlichen Nachlaß seines Großvaters zugänglich gemacht. Für Nachweise aus den Nachlässen von Hans Lewy und Elias Bickerman danken wir unseren Kollegen aus Jerusalem Guy G. Stroumsa und vor allem Albert I. Baumgarten, der uns großzügig Einblick in das von ihm im Verlauf seiner Forschungen zu Leben und Werk von Elias Bickermann zusammengestellte Material gewährte; vgl. jetzt: A. I. BAUMGARTEN, Elias Bickerman as a Historian of the Jews. A Twentieth Century Tale, Tübingen, 2010.

2 Abbildungen in B. Kytzler, K. Rudolph, J. Rüpke (Hg.), Eduard Norden (1868-1941). Ein deutscher Gelehrter jüdischer Herkunft, Stuttgart, 1994; Bildanhang, Taf. 1-8 mit Erläuterungen von J. Rüpke, S. 237-239; siehe auch E. NORDEN, Kleine Schriften zum 


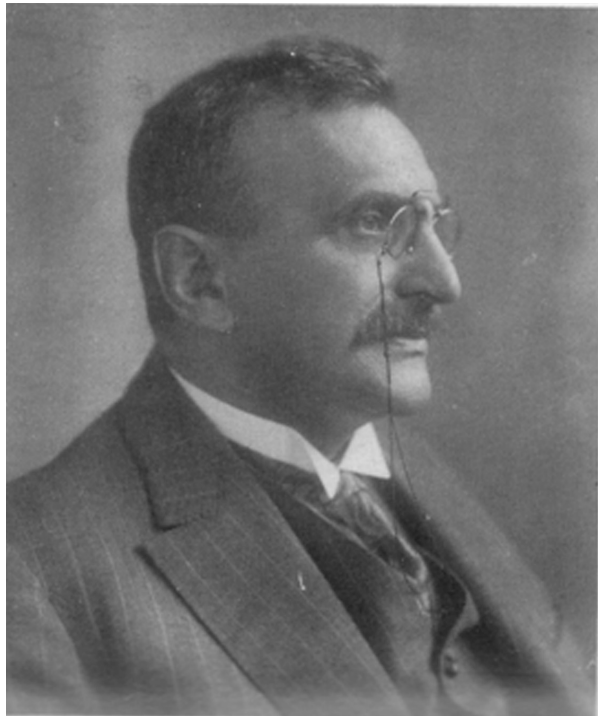

Abb. 1 Der sechzigjährige Eduard Norden

21.9.1928 wurde er sechzig Jahre alt, und dies, Nordens 60. Geburtstag, dürfte der Anlaß für dieses sehr repräsentative, aber zivile Porträt sein. Es gibt natürlich auch Bilder des Rektors im bestickten Talar, mit Barett und Amtskette (Abb. 2), sogar ein Lichtbild vom Akte der Rektorats-Übergabe selbst ${ }^{3}$. Sie wurde mit erheblichem Aufwand in der als Aula genutzten königlichen Bibliothek vollzogen. Das Gebäude wurde mitsamt dem riesigen Gemälde, vor dem die Zeremonie sich entfaltete, im Jahre 1944 zerstört. Jenes Lichtbild (Abb. 3) hat insofern nicht nur biographischen Wert. Norden, im Zentrum des Geschehens, legt den Amtseid ab, rechts und links in den Bänken stehend der Senat; vorne gemischtes Publikum; an der Wand hinten und rechts vorne salutieren korporierte Studenten in Uniform. Das Wandgemälde von Arthur von Kampf (1864-1950) zeigt Fichte bei seiner Rede an die deutsche Nation; unter seinen Zuhörern Wilhelm von Humboldt, Friedrich Schleiermacher, Henriette Herz. Da letztere nicht arischer Herkunft war, das kolossale Fresko aber nicht entfernt werden konnte, wurde es, so die Überlieferung, 1933 zugehängt ${ }^{4}$.

Die Amtseinführung, die Freude der Familie und die gesellschaftlichen Pflichten, die das hohe Amt mit sich brachte, sind in einem bemerkenswerten Dokument, den „Erinnerungen“ von Marie Norden, geschildert": „Sehr feierlich wirkt stets der

Klassischen Altertum, hg. von B. KYTZLER, Berlin 1966, Frontispiz. - Materialien zu Nordens Rektorat sind im persönlichen Nachlaß Nordens vorhanden, auch Photographien; vgl. handschriftliches Verzeichnis des persönlichen Nachlasses (Peter Norden).

3 Zur Rektoratsübergabe vgl. W. ABEL, „Studium Berolinense, 2: Eduard Norden“, Gymnasium 91 (1984), S. 449-484: zu den Jahren 1924-1931: S. 467 f.

4 RÜPKE (s. o. Anm. 2) Bildanhang Tafel 4 mit Erläuterung, aber ohne Quellenangabe für die angeführte Überlieferung. - Zu Arthur von Kampf vgl. O. Thomae, Die PropagandaMaschinerie. Bildende Kunst und Öffentlichkeitsarbeit im Dritten Reich, Berlin, 1978. - Der Kunsthistorikerin Kathrin Hoffmann-Curtius danken wir für freundliche Auskunft.

5 „Erinnerungen an Eures Vaters Leben, des Professors Dr. Eduard Norden, aufgezeichnet in Zürich von Eurer Mutter" (Manuskript p. 73-74). - Diese Erinnerungen (111 handschriftliche Seiten und 18 Seiten Nachträge), abgefaßt nach dem Tode ihres Mannes ab1942, beendet am 10.1.1944: „im 5. Kriegsjahr“, sind ein Dokument, das als solches gewürdigt werden müßte: fokussiert auf die Familie, strikt apolitisch, auch in der „sicheren Schweiz" und nach Stalingrad kein polemisches Wort zum Nationalsozialismus; selbst 


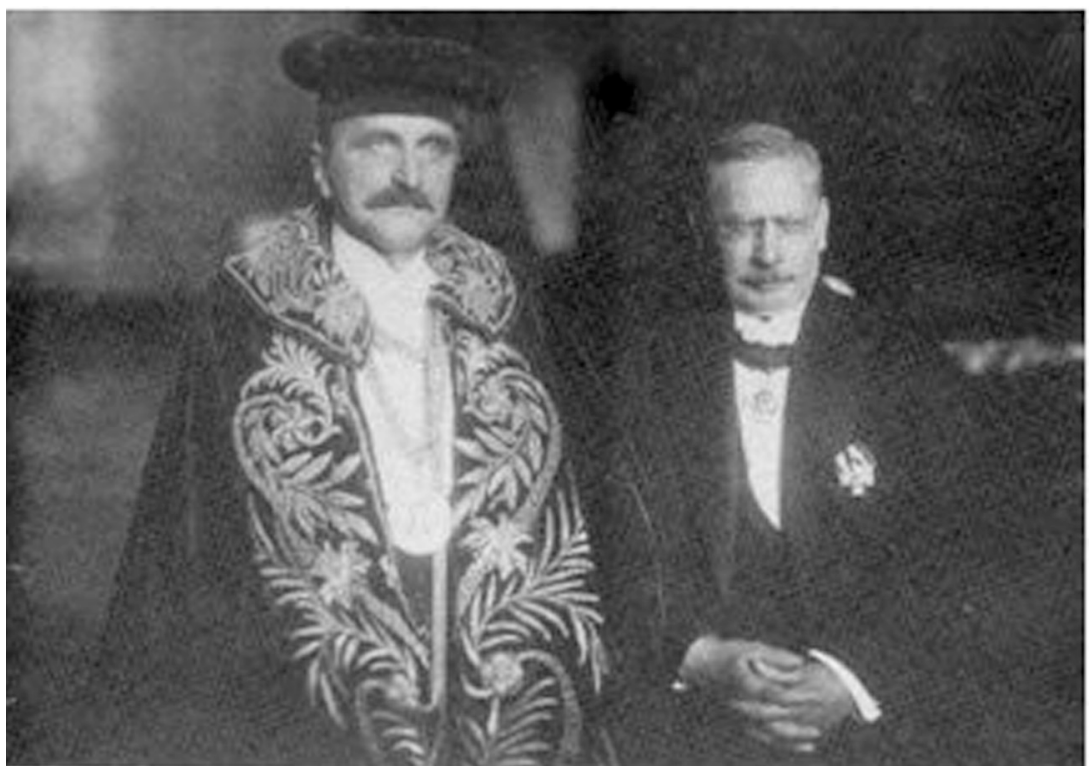

Abb. 2 Rektor Eduard Norden mit Prorektor Ernst Triepel

Augenblick, wenn der neu gewählte Rektor das Podium betritt und von den 2 Pedellen in ihrer scharlachroten Amtstracht mit dem prächtigen Rektormantel aus purpurrotem Samt mit Goldverbrämung eingekleidet wird, den der alte Rektor ablegt und mit der goldenen Halskette mit dem Bild des Königs Friedrich Wilhelm III geschmückt wird. Die Eidesleistung erfolgt in lateinischer Sprache, darauf spricht der scheidende Rektor dem Nachfolger seine Glückwünsche aus, und begibt sich in den Kreis der Collegen zurück. Eures Vaters Thema der gehaltvollen Rektoratsrede lautete: ,Logos und Rhythmus'."

Eduard Nordens Mutter versammelte trotz ihres hohen Alters „die Nordenfamilie“ zu einem festlichen Essen: „ihre 3 Söhne, der Amerikaner Hermann mit seiner Elfrieda war gekommen, Walter der Haussohn und unser Kreis mit den drei Kindern ." Abends

Hitler wird ohne Umstände erwähnt (p. 37/64). Charakteristisch sind Euphemismen und harmonisierende Umschreibungen politischer Ereignisse. Die wissenschaftliche Arbeit des Gatten hat Marie Norden, die sich „eine ungebildete Frau“ nennt, klug und geschickt durch Zeugnisse anderer kenntlich gemacht. Sie benutzt hervorragende Quellen, die alle erstaunlicher Weise aus Berlin-Lichterfelde hatten gerettet werden können.

6 Eduard Norden und seine Brüder Walter und Hermann sind Söhne des Dr. med. Carl Norden (1836-1903) und seiner Ehefrau Rosa, geb. Hamburger (geb. am 8.2.1841 in Böhmisch-Leipa, gest. am 13. Oktober 1929 in Berlin, bestattet auf dem Parkfriedhof Lichterfelde). - Eduard und Marie Norden, geb. Schultze hatten vier Kinder: Irmgard (geb. 1898), Erwin (geb. 1900), Ulrich (geb. 1903), Gerda (geb. 1909). 
wurde im ,Hotel du Nord', wie das gastliche Haus der Norden in Berlin-Lichterfelde genannt wurde, mit den Freunden Jaegers und Ed. Meyers in größerem Kreise der eindrucksvolle Tag beschlossen.

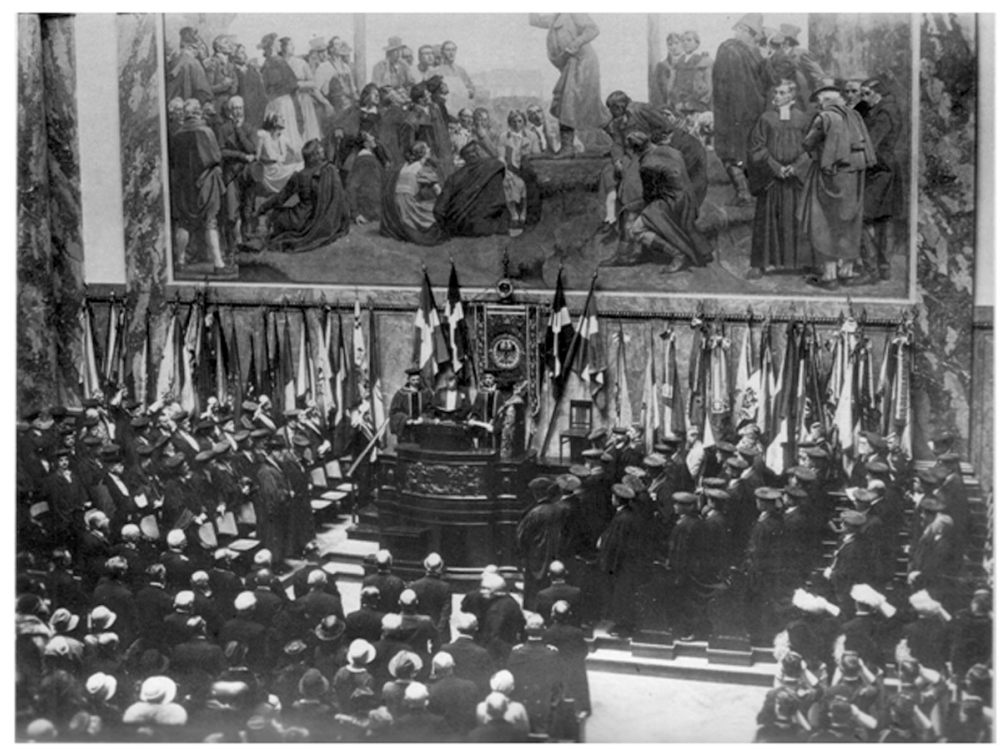

Abb. 3 Die Vereidigung des Rektors der Friedrich-Wilhelms-Universität zu Berlin am 15.10.1927

\subsection{Heldenehrungen}

In seiner Eigenschaft als Rektor hat Norden nicht nur seine Rektoratsrede über Logos und Rhythmus gehalten ${ }^{7}$. Am 3. August 1928 sprach er, bei der Feier zur Erinnerung an den Stifter der Berliner Universität, König Friedrich Wilhelm III., in der alten Aula über „Heldenehrungen bei den Griechen, Römern und alten Germanen". Der Rektor hatte reiche wissenschaftliche Vorarbeiten zum Thema und einen nahen Anlaß. Marie Norden gibt eine klare Zusammenfassung ${ }^{8}$ : „Er hat in dieser Rede als erster nachgewiesen, daß die Idee vom unbekannten Soldaten nicht in Frankreich oder England sondern in Deutschland entstanden ist. Im Jahre 1848 ist sie in Deutschland schon nachweisbar. 1866 erschien ein deutsches Gedicht über diese Frage, und 1915 kam ein Kriegsbuch heraus mit dem Titel „der unbekannte Soldat“, Verfasser Karl Bröger."

8 Erinnerungen, p. 110; vgl. p. 76. Ein Bezug zu dem „Unbekannten Gott" wird nicht hergestellt. 
Zwei Jahre zuvor, im Sommer 1926, war das Denkmal für die Universitätsangehörigen, die im Weltkrieg umgekommen waren, mit großem Aufwand im hinteren Teil des Universitätsgartens und einer Gegendemonstration im nahen Kastanienwäldchen eingeweiht worden ${ }^{9}$. Kathrin Hoffmann-Curtius und Richard Faber haben die kunstgeschichtliche, religiöse, politische Bedeutung des Ehrenmals und der Rede des Rektors dargestellt. Norden verbindet seine Ausführungen über Heldenehrungen des Altertums mit der neuen Anlage ${ }^{10}$ : „Durch die Fenster dieses Saals (der alten Aula) schweift der Blick auf das Denkmal der im Weltkrieg gebliebenen Heldensöhne unserer Alma Mater.“

Trauer, Ruhm und Rache lehren das Altertum und die famose Inschrift auf dem neuen Denkmal: invictis victi victuris. Diese Kombination von Gelehrsamkeit, Totenkult und Revanchismus ist bald nach der Niederlage im Weltkrieg, dem Sturz der Monarchie und der Einrichtung der Republik hergestellt worden. Damals, 1919, beginnt Norden einen populären Vortrag (25.11.1919) über „Bildungswerte der lateinischen Literatur und Sprache“ mit dem Satz ${ }^{11}$ : „Jede Revolution ist traditionsfeindlich“. Er schließt mit dem Aufruf ${ }^{12}$ : exoriare aliquis nostris ex ossibus ultor - „erheben soll sich aus unseren Gebeinen ein Rächer“. Ist Rache ein „Bildungswert“ des humanistischen Gymnasiums? Derartige Rhetorik war nicht ohne Wirkung. Norden erhielt Feldpostkarten seiner Studenten im Ersten Weltkrieg; einer schrieb, er sei „in

9 K. Hoffmann-Curtius, „Das Kriegerdenkmal der Berliner Friedrich-Wilhelms-Universität 1919-1927: Siegexegese der Niederlage" (mit Abbildungen und weiterführender Literatur), Jahrbuch für Universitätsgeschichte 2 (2002), S. 87-116.; DIES., „Die Sakralisierung des Kriegerdenkmals der Friedrich-Wilhelms-Universität zu Berlin. Gefallenenkult als religion civile“, in S. LANWERD (Hg.), Der Kanon und die Sinne. Religionsästhetik als akademische Disziplin, Luxembourg, 2003 (EtRellLux 2), S. 135-150. CH. SAEHrEndT, „Antisemitismus und politische Gewalt an der Berliner Friedrich-Wilhelms-Universität 1918-1933“, Jahrbuch für Antisemitismusforschung 13 (2004), S. 139-160, S. 149 ff. Die Figur des nackten sitzenden Kriegers mit Lendenschurz, Schild und Schwert hat Hugo Lederer gefertigt; vier Pfeiler begrenzen den Platz. Einweihung am 10. Juli 1826 im Beisein von Reichspräsident Hindenburg, Reichskanzler Marx, Reichswehrminister Geßler und Dr. Carl Heinrich Becker, dem Minister für Wissenschaft, Kunst und Volksbildung. Die Inschrift stammt von Reinhold Seeberg, Professor für evangelische Theologie. Sie macht Anleihen bei antiker Formensprache, wie z. B. Senecas vicimus victi.

10 E. Norden, Heldenehrungen, Berlin, 1928, gedruckt 1929, 26 S.; hier: S. 6; die Rede, um die tagespolitischen Passagen gekürzt, in Norden, Kleine Schriften [Anm. 2], S. 552-564. Die Kürzung der zeitbedingten Passagen durch den Herausgeber hat H. Haffter in seiner Rezension (Gnomon 40, 1988, S. 75-77) moniert; speziell zu diesen Passagen R. FABER, „Eduard Nordens Heldenehrungen“, in Kytzler/ Rudolph/ RüPKe (Hg.) Eduard Norden [Anm. 2], S.191-203.

11 Norden, Bildungswerte der lateinischen Literatur und Sprache auf dem humanistischen Gymnasium, Berlin 1920, in DERS. Kleine Schriften [Anm. 2], S. 583-607.

12 Vergil, Aeneis 4,625. 
den Schlachtengraus gezogen auf den Lippen tragend die Worte dulce et decorum est pro patria mori".

Norden zitiert den Fluch der rasenden Dido, die punischen Kriege, deren dritter Staat und Stadt Karthago auslöschten. Ein ominöses Zitat auch deshalb, weil sich Norden damit auf die Seite der Verlierer stellt, der Punier, des Erbfeindes Hannibal.

Im Jahre 1928 nun stand humanistisch dekorierter Patriotismus nicht mehr auf dem Programm. Rektor Norden hatte sogar, in Erwartung von Auseinandersetzungen zwischen rechten und republikanischen Studenten, Polizeischutz für die Geburtstagsfeier des Universitätsgründers anfordern müssen ${ }^{13}$.

Wenige Wochen zuvor hatten Studenten, insbesondere der nationalsozialistische Studentenbund (NSDStB), im Lustgarten so heftig gegen den Friedensvertrag von Versailles demonstriert, daß die Reichsregierung diese Kundgebung für das folgende Jahr verboten hatte. In diesem Jahre, 1929 unter Nordens Prorektorat, wird der NS-Studentenbund die zweitstärkste Gruppierung an der Universität, nach den schlagenden Korporationen ${ }^{14}$. Welche Stellung Norden in den hier angedeuteten Konflikten als Rektor oder als Bürger der Weimarer Republik bezogen hat, ist, wie es scheint, noch nicht erforscht.

\subsection{Das wissenschaftliche Cuvre um 1927/28}

Das Rektorat ist der äußere Höhepunkt einer schnellen, frühen, sehr erfolgreichen wissenschaftlichen und akademischen Laufbahn. Nordens große, innovative und arbeitsintensive Monographien liegen zu diesem Zeitpunkt bereits vor. An jede von ihnen konnten Schüler anknüpfen, die über graeco-orientalische Literatur oder Religion und jüdischen Hellenismus, über Formgeschichte und Ethnographie von Barbarenvölkern arbeiten wollten oder, unter neuartigen Bedingungen, mußten.

Schon Die antike Kunstprosa vom 6. Jahrhundert bis in die Zeit der Renaissance (1898) bezieht das Neue Testament der Christianer und christliche Autoren ein. Norden rezipiert, als einer der ersten, den Begriff „Form“ und die formgeschichtliche Methode, die Franz Overbeck entwickelt hatte ${ }^{15}$. Die Erklärung des sechsten Buchs von Vergils Aeneis, des Unterweltbuchs (1903), bringt eine Einleitung zu antiker

13 SAehrendt, „Antisemitismus und politische Gewalt“ [Anm. 9], S. 154.

14 SAEHRENDT, ebd.

15 E. Norden, Die antike Kunstprosa vom VI. Jahrhundertv. Chr. bis in die Zeit der Renaissance (1898), 2./3. Aufl. 1909/1915; Ndr. 1958, Bd. 2, S. 479 f., 492; vgl. H. CANCIK/ H. Cancik-Lindemaier, „Formbegriffe bei Eduard Norden“, in Kytzler/ Rudolph/ RüPKe (Hg.), Eduard Norden [Anm. 2], S. 47-68; FrAnz OvERBECK, Werke und Nachlaß3. Schriften bis 1898 und Rezensionen, hg. von H. CANCIK und H. CANCIK-Lindemaier, Stuttgart Weimar, 2010, S. 5-17; S. 28-30. 
Eschatologie, auch jüdischer und christlicher ${ }^{16}$. Auch Nordens dritte Monographie zielt auf das Verhältnis von orientalischer Religion und griechischer Kultur. Der Titel Agnostos Theos. Untersuchungen zur Formengeschichte religiöser Rede (1913) zitiert die Areopagrede des Paulus in der Apostelgeschichte des Lukas. Paulus diskutiert auf dem Markt in Athen mit Philosophen. Er zitiert sogar, jedenfalls in dem Bericht des Lukas,

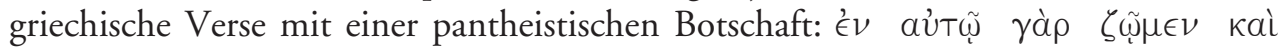

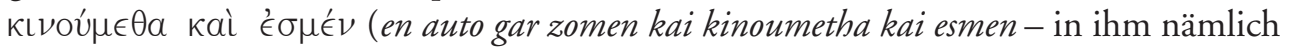
leben wir und bewegen wir uns und sind wir: Apg 17,28/ Arat, Phaen. 5). Deshalb war die Areopagrede bei christlichen Philhellenen immer beliebt. Der Untertitel der Monographie benennt Nordens wichtigste Methode: „Formengeschichte“. Norden nimmt Overbecks Programm auf ${ }^{17}$ : „Eine Formengeschichte wird also jede wirkliche Literaturgeschichte sein". Stoffwahl und Methode machten Nordens Buch für Theologen wichtig ${ }^{18}$. Für einige seiner Studenten hatte der Teil "Judaica“ eine besondere Bedeutung. Denn hier wird jüdischer Hellenismus bestimmt als eine Voraussetzung und ein Teil des Christianismos. Als Motto für das Buch aber hat Norden die Worte: "ैv tò rãv (hen to pan - Eines das Ganze) gewählt. Das Motto umrahmt die Ewigkeitsschlange, die sich in den Schwanz beißt. Das Motto ist auch ein Zeugnis für Nordens religiöses Weltgefühl (vgl. u. \$1.4).

Die evangelisch-theologische Fakultät der Universität Bonn verlieh Norden, im Hinblick auf diese Monographie, die theologische Doktorwürde ehrenhalber (3. August 1919). Noch in seinen letzten „Bestimmungen“ schreibt Norden, daß er „stolz im wahren Sinne des Wortes" eigentlich nur auf diese Ehrung gewesen sei ${ }^{19}$.

Im Jahre 1920 erschien Die germanische Urgeschichte in Tacitus Germania. Auch diese umfangreiche Monographie sucht „Formensprache“, sucht eine „Idee“, diesmal eine ethnographische, sucht Grundzüge der antiken Ethnographie: allgemeine Beschreibungsschemata, literarisch tradierte Wandermotive, Klischees, Topoi ${ }^{20}$.

16 Zur Einordnung vgl. H. CANCIK, „Libri fatales. Römische Offenbarungsliteratur und Geschichtstheologie“ (1983), in DERS., Römische Religion im Kontext. Gesammelte Aufsätze I (hg. von H. Cancik-Lindemaier) Tübingen, 2008, S. 88-114.

17 Franz Overbeck, „Über die Anfänge der patristischen Litteratur“ (1882), in OWN 3 [Anm. 15], S. 33-90; Zitat: S. 38; zum Formbegriff vgl. die Einleitung, S. 10-13; S. 28-30 über die beiden - gegensätzlichen - Stellungnahmen Nordens zu Overbecks Postulat einer christlichen „Urlitteratur“ außerhalb der antiken Literaturgeschichte.

18 Norden lehnt sich an Harnack an: S. 1 (erster Satz); S. 9 zitiert zustimmend A. Harnack, Die Apostelgeschichte, Leipzig, 1908, S. 110. Er fordert „die gemeinsame Arbeit des Theologen und Philologen".

19 Norden, Bestimmungen (1935/1940; Nachlaß).

20 K. Bringmann, "Topoi in der taciteischen Germania“, in H. Jankuhn/ D. Timpe (Hg.), Beiträge zum Verständnis der Germania des Tacitus, Göttingen, 1989, S. 59-78. - Zum damaligen Forschungsstand vgl. J. IrmsCher, „Eduard Nordens Weg zur Germanenforschung", in Rom und Germanien. Dem Wirken Werner Hartkes gewidmet, Berlin, 1983, S. 114-119 ( = SB Akademie der Wissensch. d. DDR, Gesellsch. 1982, Nr. 
Sie formen, so Nordens beunruhigender Nachweis, die Beschreibung von Barbaren, ob Skythen (Herodot, B. IV), Kelten, Germanen oder Nordvölker. Der komparatistische Formalismus wird aufgefangen von einem ausdrücklichen lokalgeschichtlichen Patriotismus: „an der Küste meiner ostfriesischen Heimat“, sagt Norden, habe er Tacitus gelesen ${ }^{21}$. Auch in der Berliner Germanenforschung hat Norden sich engagiert. Zusammen mit Dr. Albert Kiekebusch (1870-1935), dem Leiter der Fachabteilung Urund Frühgeschichte am Märkischen Museum, hat Norden 1923 den ersten Band der Reihe „Deutsche Urzeit“ herausgegeben. Seine Rektoratsrede von 1928 schließt mit germanischen Heldenehrungen und einer überraschenden Identifikation: Den Mann, den die Germanen als primum omnium virorum fortium besingen (Tac. Germania), nennt Tacitus in römischer Interpretation Hercules - auf germanisch sei es Siegfried von Xanten, der „sterkest aller Recken“ (Nibelungenlied, Vers 1671). In Nordens Euvre steht, als dritter Stoffbereich, „das Germanische“ fest neben klassischer und jüdisch-christlicher Antike ${ }^{22}$. Paradoxer Weise ist gerade diese deutsch-patriotische Arbeit zum „Vorbild“ geworden für das moderne Standardwerk, das die Zeugnisse hellenischer und römischer Autoren über Juden und Judentum sammelt und kommentiert: Greek and Roman Authors on Jews and Judaism (1974-1984) von Hans Lewy und Menahem Stern ${ }^{23}$.

Nordens fünfte große Monographie behandelt wiederum ein religionsgeschichtliches Thema: Die Geburt des Kindes (1924). Das Buch ist der Evangelisch-Theologischen Fakultät der Rheinischen Friedrich-Wilhelms-Universität zu Bonn gewidmet, als Dank für die Verleihung der Ehrendoktorwürde. Eine „religiöse Idee“ wird, ausgehend von Vergils sogenanntem „messianischen Gedicht“ (Ekloge IV), in Orient und Abendland beobachtet. Norden schließt seine komparatistische Motivgeschichte mit einem Gedicht von Gottfried Keller ${ }^{24}$ :

15); A. A. Lund, Germanenideologie im Nationalsozialismus. Zur Rezeption der „Germania“ des Tacitus im „Dritten Reich“, Heidelberg, 1995.

21 E. Norden, Die germanische Urgeschichte in Tacitus Germania, Leipzig, Berlin, 1920, Vorrede zur ersten Auflage.

22 Die philosophischen Themen seiner Habilitationsschrift hat Norden dagegen nicht weiter verfolgt.

23 Siehe Hans Lewy, Zwischenbericht p. 5, vgl. hier \$2.3.3.

24 G. Keller, „Frühlingsglaube“, in Gesammelte Gedichte, Buch der Natur Nr. 27 (= G. Keller, Sämtliche Werke, Bd. 9, Basel - Zürich, 2009). Das von Norden zitierte Gedicht ist in drei Fassungen erhalten, s. die online -Paralleldarstellung der Historisch-Kritischen Gottfried Keller-Ausgabe (HKKA): (http://www.gottfriedkeller.ch/GG/HG_parallel. htm) - (eingesehen am 2. April 2010). Norden zitiert die Fassung letzter Hand - offensichtlich aus dem Gedächtnis (s. u.). Das Gedicht ist, anders als in Nordens Wiedergabe, in fünf vierzeilige Strophen gegliedert; Norden zitiert die ersten drei Strophen. Er läßt das Zitat enden mit dem „leuchtenden Recht“ der Propheten - gemeint sind Jesaja (cap. 7 und 9, aufgenommen in der Kindheitsgeschichte des Lukas) und Vergil, vgl. Geburt des 
Es wandert eine schöne Sage

Wie Veilchenduft auf Erden um;

Wie sehnend eine Liebesklage

Geht sie bei Tag und Nacht herum.

Das ist das Lied vom Völkerfrieden

Und von der Menschheit letztem Glück;

Von goldner Zeit, die einst hienieden -

Der Traum als Wahrheit kehrt zurück - ,

Wo ewig ${ }^{25}$ alle Völker beten

$\mathrm{Zu}^{26}$ einem König, Gott und Hirt;

Von jenem Tag, wo den Propheten

Ihr leuchtend Recht gesprochen wird.

Auch dieses Zitat dürfte als Selbstzeugnis zu verstehen sein.

Nach seinem Rektoratsjahr hat Norden noch zwei Monographien veröffentlicht, beide setzen ältere Arbeiten fort. Eine Nachlese zu der großen ethnographischen Studie über Tacitus' Germania bietet sein Altgermanien (1934). Die Stoffwahl paßte gut zu dem völkischen Enthusiasmus nach dem Ende der Weimarer Republik. Die letzte, unter immer größeren Schwierigkeiten geschaffene und schließlich, nachdem deutsche Verleger sich versagt hatten, in Schweden gedruckte Veröffentlichung Aus altrömischen Priesterbüchern (Lund 1939) hat eine lange Entstehungsgeschichte. Sie beginnt, wie Jörg Rüpke in detaillierter Werkanalyse mit wissenschaftsgeschichtlicher und biographischer Absicht gezeigt hat, mit den „Altlateinischen Studien“ in den 20er Jahren ${ }^{27}$. Auch hier werden Formeln untersucht, Formen, Formulare, offiziöse Urkunden der Auguren und Ackerbrüder der Stadt Rom. Norden liefert eindrucksvolle Beiträge zur Religions-Philologie, fixiert die frühe, sozusagen ursprüngliche Verbindung von griechischer mit römischer Religion und Literatur, also gerade nicht den erwarteten altlateinisch-indogermanischen Ursprung römischer Kultlieder.

Nordens letzter Berliner Plan ist überschrieben: „Heimatkundliches zu Horaz“; der weit gesteckte Rahmen ist eine Darstellung der lateinischen Literatur „unter dem

Kindes, S. 21. Warum er die Strophengliederung unterdrückt hat, ist uns nicht erklärlich, ebenso wenig die beiden Gedankenstriche.

„Wo ewig“ ...- Keller: „Wo einig“ “..

26 „Zu einem König“ ... - Keller: „Zum Einen König“ ...

27 J. RüPKE, Römische Religion bei Eduard Norden, Marburg, 1993; in den Anhängen finden sich die Briefe Nordens an M. P. Nilsson (1920-1939) und eine Übersicht über Nordens Lehrveranstaltungen (zu letzteren vgl. W. A. SCHRÖDER, Der Altertumswissenschaftler Eduard Norden (1868-1941), Hildesheim, 2001 (Spudasmata 73), S. 185-188). Siehe auch RÜPKE, „Der späte Norden (1925-1941): Die Entstehung der ,Altrömischen Priesterbücher" als biographischer Schlüssel“, in KytZleR/ Rudolph/ Rüpke (Hg.), Eduard Norden [Anm. 2], S. 129-150. 
Gesichtspunkt der Stämme und Landschaften ihrer Vertreter ${ }^{28 “}$. Norden zitiert mit dieser Themenstellung die Vision einer „Literaturgeschichte der deutschen Stämme und Landschaften", die Josef Nadler seit 1912 verbreitet hatte ${ }^{29}$. Also nicht mehr Konstruktionsprinzipien von Kunstprosa, das Verhältnis von religiöser Idee und sprachlicher Form, die intricate Vermischung von morgen- und abendländischen Traditionen, die Geburt des Christentums aus dem jüdischen Hellenismus, sondern ein betont völkisches Programm. Dieser letzte Arbeitsplan, den Norden 1935 der Akademie vorlegte, kann als Anpassung an systemnahe Konzepte verstanden werden. Den freiwilligen Austritt aus der Akademie, zu dem er am 12.10.1938 gezwungen wurde, konnte dieser Plan jedenfalls nicht verhindern. Es muß berücksichtigt werden, daß dieser letzte ein alter Plan war. Wie die Studien zu Altgermanien (1934) und den altlateinischen Sakralformeln (1939) wurde er in Nordens frühen Berliner Jahren entwickelt. Bereits in seiner Antrittsrede im Jahre 1913 sprach Norden vor der Berliner Akademie über "Wechselwirkung von Landschaft und geistigem Leben aufeinander", er wollte einen "geographisch-ethnographischen orbis litteratus" aufbauen, eine Art regionale Literaturgeschichte, und schon damals existierten dafür Sammlungen von einem ,ziemlich großen Umfang 30 “.

Der letzte Plan Nordens zeigt zweierlei: Verzicht auf neue wissenschaftliche Ansätze und Pläne bzw. kluges Anknüpfen an vorhandene Vorarbeiten und Anpassung des Konzeptes einer regionalen Literaturgeschichte an völkische Begrifflichkeit. Nordens Arbeitsbedingungen verschlechtern sich jedoch so schnell und einschneidend, daß an eine Ausführung des Plans nicht zu denken war. Norden mußte im Januar 1938 Teile seiner Bibliothek und sein Haus in Berlin-Lichterfelde verkaufen. Im Oktober 1938 folgte, wie erwähnt, der Zwangsaustritt aus der Akademie, am 8.12.1938 die Anweisung des Ministeriums, die Nicht-Ariern den Zutritt zur Universität und ihren Einrichtungen untersagte. ${ }^{31}$

28 Nordens Vortrag in der Berliner Akademie am 4.3.1935. Resumé des Vortrags in den Sitzungsberichten der Akademie, zitiert nach E. MEnsCHING, Nugae zur Philologiegeschichte 1, Berlin 1987, S. 63.

29 J. NADLER, Literaturgeschichte der deutschen Stämme und Landschaften. 1. Die Altstämme (800-1600), Regensburg 1912. Vgl. I. RanZmaier, Stamm und Landschaft: Josef Nadlers Konzeption der deutschen Literaturgeschichte, Berlin u. a. 2008.

30 Norden, Antrittsrede in der Berliner Akademie der Wissenschaften, 1913, in Kl. Schr., S. 676-677; ein Bezug auf Nadler ist nicht expliziert; Norden sucht lokale Zentren, territoriale Einheiten, regionale Literaturgeschichte und zwar für den ganzen antiken Mittelmeerraum, nicht nur für Italien.

31 Die Liste der Repressalien ist nicht vollständig; weiteres bei SCHRÖDER, Der Altertumswisssenschaftler [Anm. 27], S. 31-49: „Die Berliner Jahre von 1933 bis zur Emigration nach Zürich (1939)“. - Das Nachwort von Ruth Mövius zu MAX Herrmann, Die Entstehung der berufsmäßigen Schauspielkunst (1933/1942), Berlin, 1962 (Übertragung der Zitate aus dem Griechischen und Lateinischen von Dr. Leiva Petersen) bringt die bedrückenden Details einer perfekten, allgemeinen, unmenschlichen Praxis 


\subsection{Die Eduard-Norden-Siftung: „,jede Confession“}

Gerade in seinem Rektoratsjahr feierte Norden seinen sechzigsten Geburtstag (21. September 1928). Ein Comité seiner nächsten Kollegen beschloß, Geld zu sammeln; Künstlerhand sollte die Züge des Gelehrten in Büste oder Gemälde festhalten. Zahlreiche Professoren der philosophischen und theologischen Fakultäten, Studienräte, Studiendirektoren - keine Firmen, Künstler, Politiker, Vereine - aus Deutschland, einige aus Polen (St. Witkowski, Lwow; Th. Zielinski), Schweden (Carlsson, Löfstedt, Sjögren, Svennung) spendeten 5. 257,10 Reichsmark. Ein einziger ungenannter Spender erhöhte die Summe auf 10.000 Reichsmark ${ }^{32}$. Die Spendenliste bietet mehr als dreihundert Namen mit Titel und Adresse, etwa Otto Weinreich und Josef Vogt aus Tübingen, Eduard Fraenkel, Hermann Fränkel, Paul Friedländer, Victor Ehrenberg, Paul Maas, Georg Rohde (vgl. Anhang 2). Den Arbeitsausschuß bildeten A. Giesecke (Teubner-Verlag), F. Jacoby, W. Jaeger.

Eduard Norden jedoch verweigerte die Herstellung eines Bildes „absolut ${ }^{33 “}$. Er errichtete vielmehr die „Eduard-Norden-Stiftung“, „bestimmt für Studenten und jüngere Gelehrte jeder Confession ${ }^{34 “}$. Die Geschichte dieser Stiftung ist noch nicht untersucht. Sie wurde, um die Herkunft zu verschleiern, in „Stiftung für classische Philologie“ umbenannt und nach Nordens „Fortgang" in die Schweiz von Johannes Stroux und Ludwig Deubner geleitet.

Die Bestimmung für ,jede Confession“ öffnet die Stiftung nachdrücklich für Studenten, die sich zum "mosaischen Glauben“ bekannten. Diese Offenheit oder Neutralität gründet in Nordens Wissenschaftsverständnis und ist vereinbar mit dem wenigen, was bisher über seine Religiosität bekannt ist. Edgar Bauer hat sie zutreffend in das weite Feld des Kulturprotestantismus eingeordnet, ihre Distanz zu jedem Fideismus und die quasi-pantheistischen, humanistischen und nationalbezogenen Komponenten bestimmt ${ }^{35}$. Der Rahmen ist das öffentliche Christentum, das die Feste im Lebenszyklus mit Eheschließung, Taufe der Kinder und Enkel, Konfirmation auszeichnet ${ }^{36}$. Die Verehrung von Werk und Person Goethes, das Ëv tò mãv (hen

von Verwaltung, Studentenschaft und Gesellschaft in Berlin zum Ausdruck; vgl. die Rezension von M. Fuhrmann, Gnomon 36 (1964), S. 537 ff. Herrmann, ein Kollege von Norden, bezeichnet sich als „nationaldeutsch“.

32 Es ist der Bruder, Hermann Norden, Paris, 22 Rue Washington. - Im Jahre 1938 beträgt das Stiftungskapital etwa RM 13.000; Verwaltungsstelle ist die Berliner Universität (Eduard Norden, Personalia, Berlin 1938).

33 Marie Norden, Erinnerungen, S. 77.

34 Ebd.

35 E. Bauer, „Eduard Norden: Wahrheitsliebe und Judentum“, in Kytzler/ Rudolph/ RüPKE (Hg.), Eduard Norden [Anm. 2], S. 205-223, bes. S. 209.

36 Haustaufe, Feiern in der Lichterfelder Paulus-Gemeinde am Hindenburgdamm, Bestattung auf dem Parkfriedhof Lichterfelde sind bei Marie Norden knapp verzeichnet. 
to pan), der „unbekannte Gott“, das Unendliche, Überwältigende, Unnahbare, das Norden immer wieder in Naturerlebnissen am Meer und im Gebirge sucht, sind nicht Rankenwerk und bildungsbürgerlicher Zierrat, sondern notwendiger Bestandteil dieses Typus von Christentum in der neueren Religionsgeschichte Deutschlands.

In der Einsamkeit des Großglockner-Gebiets, in der Hoffmannshütte versucht Norden, seinen Eindruck von der Gletscherlandschaft in Worte zu fassen ${ }^{37}$ :

Die Natur ist so überwältigend, dass man ohne Maler zu sein, freilich gar nicht wagen darf sie zu entweihen durch Worte und Federstriche <.> Todeseinsamkeit inmitten einer Gletscherwelt; urweltliche Ewigkeiten; nach Westen zu nur Eis und Schnee, in direkter Linie mir gegenüber südlich der Glockner mit seinen beiden Spitzen; nach Osten zu nackter Fels, nur hie und da noch durch etwas Grün unterbrochen. Kein Sterbenslaut, nur der heulende Wind u. hin und wieder ein donnerartiges Getöse, von „Eisbruch“ herrührend. Aber wie gesagt ich verzichte das auszuführen. „Majestätisch“ träfe das noch nicht, vielleicht „Titanisch“, aber man fühlt sich doch der Unnahbarkeit Gottes um einen Fussbreit näher gerückt. Vielleicht würde mancher Atheist, der zeitlebens in der Ebene lebt, hier zum Bekenner eines göttlichen Urwesens werden können. [...] jetzt zum ersten Male fühle ich das Unnahbare mir nahe gerückt $u$. doch ist das, was jetzt um mich ist, doch nur wieder ein Abglanz dessen, das diejenigen schauen dürfen, die nicht durch die Erbärmlichkeit ihrer somatischen Materie gezwungen sind mit, wenn auch noch so kostbaren Surrogaten, vorlieb zu nehmen, sondern die noch tiefer in diese Majestät des Todes und des urweltlichen Schweigens eindringen können.

Um derartige „Eindrücke“ auch einem akademischen Lesepublikum zu vermitteln, stellt er Gottfried Kellers „Frühlingsglaube“ an den Schluß seiner Schrift Die Geburt des Kindes und Verse von Wilhelm Raabe an den Schluß seiner Rektoratsrede über Logos und Rhythmus ${ }^{38}$ :

Das Ewige ist stille,

Laut die Vergänglichkeit.

Schweigend geht Gottes Wille

Über den Erdenstreit.

In seinen letzten „Bestimmungen“ ordnet Norden an, „der wesentliche Teil“ der Ansprache des Geistlichen - nur dieser sollte bei seiner Beisetzung sprechen - möge

37 E. Norden, 1911, in Marie Norden, Erinnerungen, Nachträge p. 4-5 (Hs.).

38 Norden, „Logos und Rhythmus“, in Kleine Schriften [Anm. 2], S. 533-551. - W. RaABE, Sämtliche Werke. Braunschweiger Ausgabe, Bd. 20, Göttingen, 1968, S. 388 f. und Anmerkungen S. 524. Das Gedicht ist überschrieben: „Stille der Natur" und laut Tagebuch am 17. 12. 1861 verfaßt. Norden zitiert die dritte von neun vierzeiligen Strophen. Diese dritte Strophe wurde in das „Thüringer evangelische Gesangbuch “ aufgenommen (Mitteilungen der Raabe-Gesellschaft 1928, S. 80). 
sich „aus eindrucksvollen Bibelstellen“ zusammensetzen ${ }^{39}$. Danach, zum Abschluß dieser „Bestimmungen“ zitiert er erneut Raabes Verse.

\section{Von Berlin nach Jerusalem}

\subsection{Altertumskunde an der Hebräischen Universität zu Jerusalem}

In dem Jahre, in welchem Eduard Norden in Berlin Rektor der zukünftigen Humboldt-Universität ist, unter Polizeischutz am 3. August den Gründer der Universität, König Friedrich Wilhelm ${ }^{40}$, erinnert, werden in Jerusalem die "Geisteswissenschaftliche Fakultät“ und „das Fach Altertumskunde“ gegründet. Die Gründungsväter heißen: Max Schwabe, Victor Tcherikover und, wenige Jahre später, Hans Lewy. Alle drei waren Studenten, Doktoren, Privatdozenten der Friedrich-Wilhelms-Universität, Schüler von Eduard Norden, Eduard Meyer, Ulrich Wilcken, Hermann Diels, Adolf Harnack, Ulrich von Wilamowitz-Moellendorff. Auf den Grundlagen, die diese drei Gelehrten unter schwierigsten Bedingungen geschaffen haben, steht auch heute noch die Altertumswissenschaft an der Hebrew University Jerusalem. Die Historikerin Hannah Cotton bestimmte deren Aufgabe folgendermaßen: ${ }^{41}$ Die „Altertumskunde“ der Geisteswissenschaftlichen Fakultät in Jerusalem erforscht „die klassische Zivilisation als Ganze; klassische Philologie, Literatur, Geschichte, Archäologie, Kunst, Papyrologie, Epigraphik, Recht, Wissenschaftsgeschichte". Cotton fährt fort: "this broad view is directly inherited from our,founding fathers', so to speak, the first classicists at the Hebrew University, who were all trained in Berlin in the tradition of German scholarship of the nineteenth and early twentieth centuries - a tradition of the integral nature of Altertumswissenschaft".

Hannah Cotton bezieht sich hierbei auf das Forschungsprogramm, das Max/ Moshe Schwabe im Jahre 1927 in deutscher Sprache formuliert hatte: ${ }^{42}$ „Memorandum über Stellung und Aufgaben der klassischen (griechischen) Altertumswissenschaft innerhalb der Hebräischen Universität Jerusalem." Das Memorandum zielt darauf,

39 Norden: Bestimmungen für meine Bestattung (bez. Cremation), Bln-Lichterfelde, 16. Okt. 1935; erweitert: Zürich, 2. Nov. 1939; 22. Nov. 1940 (Nachlaß).

40 Friedrich Wilhelm, (*3. August 1770 in Potsdam, † 7. Juni 1840 in Berlin) war seit 1797 König von Preußen.

41 Ansprache anläßlich des Geburtstages von Prof. Fikhman und der Vorstellung seiner Kleinen Schriften in Heidelberg, 2006 (freundliche Mitteilung von Hannah Cotton). Vgl. I. F. Fikhman, Wirtschaft und Gesellschaft im spätantiken Ägypten: Kleine Schriften, hg. von A. Jördens, Stuttgart, 2006 (Historia. Einzelschriften, H. 192).

42 Publiziert bei R. Meridor und J. Geiger, „The Beginnings of Classics in Israel: Two Documents“, Scripta Classica Israelica 18 (1999), S. 159-173. - Das Programm berücksichtigt nicht die Aus- und Weiterbildung von Lehrern. 
das Berliner kulturwissenschaftliche Modell an die besonderen Möglichkeiten und Schwierigkeiten in Palaestina anzupassen. Zur Begründung führt Schwabe aus: „Der gemeinsame Untergrund aller westlichen Kulturen ist ein griech. röm. humanistischer, dessen Kenntnis und Verständnis für uns mithin eine organische Notwendigkeit ist".

Die Anpassung, die Schwabe vornimmt, setzt den Schwerpunkt auf den Hellenismus und die Kaiserzeit, auf den östlichen Teil der méditerranée und Vorderasien, auf Materialkunde vor Ort (Epigraphik, Papyri), auf jüdische Geschichte - all dies zu Lasten der archaischen und klassischen Autoren und Orte; also: Antiochia am Orontes und das ägyptische Alexandria, nicht Mykene und Athen. Das altertumswissenschaftliche Modell, das auf Sprache und Realien, materieller Kultur, Recht und Religion, Urkunden und Inschriften insistierte, erwies sich für jüdische Geschichte in den Diadochenreichen und der römischen Provinz Syrien-Palaestina als besonders anpassungsfähig.

Eine vergleichbare Aufbauarbeit hat ein anderer "Schüler" Nordens an anderem Orte geleistet: Georg Rohde (1899-1960). Er mußte, da er nicht arisch genug verheiratet war, Deutschland verlassen; Norden hat seinen Neuanfang durch Gutachten unterstützt. Von 1935 bis 1950 hat Rohde in Ankara die Klassische Philologie aufgebaut, die Bibliothek eingerichtet, Übersetzungen ins Türkische geschaffen, einen großen türkischen Schülerkreis betreut ${ }^{43}$.

\subsection{Elias Bickermann, geboren 1897 in Kishinew, gestorben 1981 in Tel Aviv}

Als für Eduard Norden wissenschaftliche Arbeit und bald auch die schiere Existenz in Berlin unmöglich wurden, hatten seine nicht-arischen Studenten bzw. "Schüler" die Stadt längst verlassen. Sie waren jünger und weniger von Familie und Vermögen belastet.

Elias Bickermann lebte seit 1922 in Deutschland ${ }^{44}, 1926$ wurde er bei Ulrich Wilcken und Norden promoviert, 1930 habilitierte er sich über die Geschichte des griechischen Hypomnema in Ägypten an der Philosophischen Fakultät der Friedrich-WilhelmsUniversität. Von 1930-1933 war er daselbst Privatdozent für Alte Geschichte ${ }^{45}$. Er war einer unter vielen. Wilamowitz hatte bereits am 5.4.1919 an Norden geschrieben ${ }^{46}$ :

43 Vgl. die Gedenkrede von Paul Moraux, in G. Rohde, Studien und Interpretationen zur antiken Religion und Geschichte, hg. von I. Rohde und B. Kytzler, Berlin, 1963, S. 4-7.

44 A. I. Baumgarten, Elias Bickerman [Anm. 1] S. 18-24: „Basic Biography“.

45 Privatdozentur von 1.3.1930 bis 2.9.1933.

46 Wilamowitz an Norden, in W. M. CALdER III / B. Huss (Hg.), „Sed serviendum officio... “. The Correspondence between Ulrich von Wilamowitz-Moellendorff and Eduard Norden, Hildesheim 1997, nr. 188 (S. 178). - Vgl . Baumgarten, Bickerman [Anm. 1], S. 99-108: "Jews in the Academy in the Weimar years", und die dort angegebenen Arbeiten, bes. A. PAWLICZEK, Wissenschaftlicher Alltag und Elitenauslese. Jüdische Dozenten an der FWU zu Berlin 1871-1933, Diss. HU Berlin (bei Rüdiger vom Bruch), im Druck. 
„Daß wir so viele Dozenten haben, alle Juden beinahe, ist nicht angenehm, aber wie ich den Pr. Doz. ansehe, ist nur die moralische und wissenschaftliche Qualität maßgebend." Nicht alle an der Friedrich-Wilhelms-Universität waren dieser Meinung. Im September 1933 wurde auch Bickermann die Lehrerlaubnis entzogen, und schon im Dezember war er in Paris und floh von dort über Marseille - Casablanca in die USA. Er nahm mit sich eine Würdigung des Rektors Eduard Norden, eine von Norden korrigierte Arbeit zu Tacitus, die Didaktik und Methodik seiner Berliner Lehrer und das treibende Moment all seiner wissenschaftlichen Arbeit.

Er zeigt Lichtbilder am Ende seiner Vorlesung an der Columbia-University in New York. Seine Maxime: „I teach you to read slow". Das treibende Moment ist die Frage, wie Athen und Jerusalem zusammen kommen könnten, die Akademie Platos mit der Kirche Christi. Die Frage ist anschaulich in der Schlüsselszene der Apostelgeschichte: Paulus auf der Agora und dem Areopag in Athen. Dies ist, nach Albert Baumgarten, die Kernfrage des gesamten wissenschaftlichen Euvres von Elias Bickermann, und sie ist entwickelt an den Beobachtungen und Fragen, die sein Lehrer Eduard Norden in Agnostos Theos und Geburt des Kindes veröffentlicht hatte.

Anläßlich der Rektoratsübergabe an Eduard Norden erschien in den „Berliner Hochschulnachrichten" (Oktober 1927, 1. Heft) eine nur mit den Initialen E. B. gezeichnete Würdigung Nordens: „E. N. Vom wissenschaftlichen Wirken des neuen Universitätsrektors". Sie ist, soweit wir sehen, in der Norden-Forschung noch nicht genutzt worden. Wir verdanken die Kenntnis dieses Artikels und weiterer Materialien zu Eduard Norden der Freundlichkeit von Bickermanns Schüler Albert Baumgarten, Althistoriker der Universität Bar Ilan. Baumgarten wird, so ist zu hoffen, die Texte alsbald veröffentlichen und erläutern ${ }^{47}$. Die Würdigung Nordens in den „Hochschulnachrichten“ wird umrahmt von Artikeln über „Farbstudententum und Alkoholfrage“ und über „Das neue Studentenrecht ${ }^{48 “}$. Ihre ersten Sätze lauten: „Eduard Norden ist Philologe. Zunächst in unmittelbarer Bedeutung des griechischen Begriffes; er liebt das Wort an sich, den Klang der schönen Rede, den sinnvollen Ausdruck. Die ihn am 15. Oktober hörten, werden sich erinnern, wie rhythmisch und gemessen, mit sichtbarer Freude am schönen Ton des feierlichen lateinischen Spruches er den Amtseid las. Seine Rede, die darnach folgte ${ }^{49}$, war auch eine rhetorische Leistung, zugleich aber zeigte sie den neuen Rektor von der anderen Seite seines

47 Vgl. A. I. Baumgarten, „Eduard Norden and his Students: A Contribution to a Portrait, Based on Three Archival Finds“, Scripta Classica Israelica 25 (2006), S. 121-139.

48 Kultusminister Carl Heinrich Beck (parteilos) versuchte, mit der „Verordnung über die Bildung von Studentenschaften" den zunehmenden Druck republikfeindlicher Organisationen einzuschränken. - Vgl. SAEHrEndT, „Antisemitismus und politische Gewalt" [Anm. 9].

49 E. Norden, Logos und Rhythmus. Rede zum Antritt des Rektorats der Friedrich-WilhelmsUniversität in Berlin, am 15. Oktober 1927 gehalten, Berlin, 1928 (= Kleine Schriften [Anm. 2] S. 533-551). 
philologischen Temperaments.“ - Der Schluß der Würdigung lautet: „Soviel - nicht von den Büchern - (es wäre dann noch vieles zu nennen), sondern von der wissenschaftlichen Persönlichkeit, wie sie sich in den Büchern erkennen läßt. Vom Menschen und Lehrer zu sprechen verbietet die Ehrfurcht vor dem Lebenden. E. B.“

E. B., das ist Elias Bickermann, der gerade bei Ulrich Wilcken und Eduard Norden mit einer 38 Seiten langen Dissertation über das Bürgerrechtsedikt Caracallas promoviert worden $\operatorname{war}^{50}$. In dem offiziösen Lebenslauf, den er in deutscher Sprache seiner Dissertation beifügte, schreibt Bickermann mit einem gewissen Nachdruck ${ }^{51}$ : "Ich bin jüdischer Abstammung und bekenne mich zum mosaischen Glauben“. Aus diesem Grunde mußte E. B. bald darauf aus Deutschland fliehen, über Paris nach New York, aber er hatte Norden im Gepäck, auch jene Würdigung anläßlich der Rektoratsübernahme.

In einer Liste von "Schülern“ Eduard Nordens dürfte, wie immer der Begriff „Schülerschaft“ bestimmt wird, der Name Elias Bickermann nicht fehlen. In der „Liste der unter Nordens Mitverantwortung entstandenen Dissertationen " muß der Name E. B. hinzugefügt werden ${ }^{52}$. Bickermann verstarb 1981 während einer Reise in Israel und ist in Jerusalem begraben.

\subsection{Max Schwabe, Victor Tcherikover, Hans Lewy}

2.3.1 Max (Moshe) Schwabe, geboren1899 in Halle, gestorben1956 in Jerusalem

Max (Moshe) Schwabe war bereits bei der Gründung der Hebräischen Universität (HUJ) 1925 in Jerusalem und Lehrer für griechische Sprache. Er war mit einer Dissertation über Libanios von Antiochien bei Hermann Diels und Ulrich von Wilamowitz-Moellendorff promoviert worden ${ }^{53}$. In der Vita, die der Arbeit angehängt ist, nimmt Norden sozusagen den Platz des Zweitgutachters ein:

Quibus cunctis (scil. magistris) imprimis Wilamowitzio et Nordeno, gratias ago candidas... Quantum his viris doctissimis debeam hic dicere si vellem vix possem.

50 E. Bickermann, Das Edikt des Kaisers Caracalla in Pap. Giss. 40, Diss. Berlin, 1926.

51 Konfessionsangaben in anderen Viten sind knapper.

52 In dieser im Anhang zu Nordens Kleinen Schriften [Anm. 2] (S. 689-690) abgedruckten Liste, „zusammengestellt von stud. Phil. Manfred Weißhaupt“, fehlt der Name.

53 M. Schwabe, Analecta Libaniana, Diss. Berlin (1914), 1918. De dissertatione probanda ad ordinem rettulerunt $U$. de Wilamowitz-Moellendorff, $H$. Diels. Die Arbeit ist Udalrico de Wilamowitz-Moellendorff gewidmet. Caput I: De nymphaeo Antiocheno; Caput II: De oratione pro Olympio habita;...; Caput IV: De Libanio psalmorum imitatore; Caput V: Observationes criticae. - M. Schwabe war dem Herausgeber des Libanios, R. Foerster, bei der Fahnenkorrektur behilflich. - Rigorosum: 28. Mai 1914; Tag der Promotion: 15. Oktober 1918. - Ein besonderer Dank am Ende der Vita gilt E. Täubler. 
Schwabe sollte, wie Bickermann, in eine Liste von "Schülern“ Nordens aufgenommen werden ${ }^{54}$.

Schwabe hat 1927, anläßlich der Gründung des Faches „Altertumskunde“ an der Geisteswissenschaftlichen Fakultät der HUJ, das Forschungsprogramm formuliert (\$2.1). Im Rektoratsjahr Nordens wurde er Lecturer für griechische Sprache und Literatur an der HUJ. Er gilt, mit den etwas jüngeren Kollegen Hans Lewy und Victor Tcherikover, als Gründungsvater der Klassischen Altertumswissenschaft in Israel. Deshalb hängen die Bilder dieser Berliner Gelehrten im Seminarraum des Classics Department der Hebrew University, Jerusalem. ${ }^{55}$.

2.3.2 Victor (Avigdor) Tcherikover, geboren 1894 in St. Petersburg, gestorben 1958 in Jerusalem

Victor Tcherikover, der zweite Gründungsvater der Klassischen Altertumswissenschaft in Israel, ist Schüler von Ulrich Wilcken und Eduard Meyer; eine Beziehung zu Norden haben wir nicht gefunden. Tcherikover hatte bei Wilcken und Meyer eine Dissertation über „Hellenistische Städtegründungen in Kleinasien“ verfaßt ${ }^{56}$. Nach der Promotion im Jahre 1925 ging er sogleich nach Palaestina und wurde Research Fellow an der neu gegründeten HU Jerusalem für das Fach Alte Geschichte. Mit Schwabe und Lewy, den Vertretern von Griechisch und Latein, bildete er 1933/34 den Lehrkörper für die inzwischen zum ordentlichen Hauptfach aufgestiegene „Klassische Altertumskunde ${ }^{57 “}$. Der Schwerpunkt von Tcherikovers Arbeit ist Papyrologie und jüdischer Hellenismus. Die drei Altertumswissenschaftler planten eine Sammlung der antiken Quellen zur Geschichte der jüdischen Siedlung in Palaestina in der Reihe Sefer

54 Worin Nordens Hilfe bestand, ist nicht angegeben. Norden, Agnostos Theos, ist in Hinsicht auf die Partizipialform von Gottespraedikationen (S. 60) zitiert; auffällig der Ausdruck Christiana superstitio für die von Konstantin tolerierte Religion (S. 61). Libanios habe, so Schwabe, die alttestamentlichen Zitate aus christlicher Tradition.

55 Schwabe steht in Briefkontakt mit Ernst Grumach (1902-1967). Grumach ist Altphilologe und Goetheforscher; 1929-1933 Lektor der Altphilologie an der Universität Königsberg, 1937-1942 Dozent an der Lehranstalt für die Wissenschaft des Judentums in Berlin; seit 1948 Professor für Klassische Philologie an der Humboldt-Universität; 1949 -1959 Herausgeber der Goethe-Ausgabe der Akademie in Berlin-Ost. - Ernst Grumach an Schwabe, 15.2.47 (Teilabdruck: G. Scholem, Briefe I. 1914-1947, hg. von I. SHEDlETzKy, München, 1994, S. 455). Grumach hat von Schwabe erfahren, daß Scholem gut aus Deutschland zurückgekehrt ist; anscheinend liegt ein Brief Grumachs an Scholem dabei, den Schwabe übergibt; Scholem antwortet am 24.4.1947 (= Brief nr. 137, S. 326).

56 Erweiterte Fassung publiziert unter dem Titel: „Die hellenistischen Städtegründungen von Alexander dem Großen bis auf die Römerzeit", in Philologus Supplement-Band XIX, 1, 1927. - Vgl. Baumgarten, Bickerman [Anm. 1], S. 190-193. Tcherikover war Zionist.

57 Hebrew University (Die Hebräische Universität), Jerusalem 1939, S. 54. Als Forschungsaufgabe wird hier genannt: „Hellenistische Epoche und Geschichte der Juden zur Zeit des 2. Tempels“, als Lehrgebiet: Alte Geschichte (Griechenland und Rom). 
ha-Jischuw. ${ }^{58}$ Der Plan der Reihe, das Thema "Siedlung“ zeigt, daß die drei Berliner Doktoranden und Privatdozenten auch im Britischen Mandatsgebiet Palaestina keinen politik- und konfliktfreien Raum gefunden hatten.

2.3.3 Hans (Johanan) Lewy, geboren1901 in Berlin, gestorben 1945 in Jerusalem

Hans Lewy war - wie Elias Bickermann, Friedrich Solmsen und Richard Walzer - von den Rassegesetzen des Jahres 1933 betroffen ${ }^{59}$. Lewy verließ Deutschland sofort und wurde noch im Jahre 1933, unterstützt durch ein Gutachten von Eduard Norden, zum "Instructor" (Senior Assistant) für lateinische Sprache und Literatur an der HUJ bestellt. Er war im Jahre 1926 mit der Arbeit Sobria ebrietas. Untersuchungen zur Geschichte der antiken Mystik promoviert worden ${ }^{60}$. Im Vorwort schreibt Lewy: „Meinem Lehrer Eduard Norden, der das Werden der Arbeit mit ständigem Interesse verfolgte und durch Anregungen förderte, sowie Werner Jaeger, der mich auf die Beziehungen des philonischen Begriffs zu den hermetischen Schriften hinwies, bin ich zu besonderem Dank verpflichtet. Wie stark diese Untersuchung von Nordens, Reitzensteins und Boussets bahnbrechenden Forschungen bestimmt ist, braucht dem Kenner der modernen religionswissenschaftlichen Forschung nicht gesagt zu werden." In seinem Lebenslauf nennt Lewy Eduard Norden, Ulrich von WilamowitzMoellendorff und Eduard Meyer „meine Lehrer ${ }^{61 “ . ~ A u c h ~ H a n s ~ L e w y ~ m u ß ~ i n ~ d i e ~}$ Liste der „Schüler“ bzw. der „unter Eduard Nordens Mitverantwortung erschienenen Dissertationen" aufgenommen werden ${ }^{62}$.

Im Juli 1933 habilitierte sich Lewy an der Philosophischen Fakultät für das neu eingerichtete Lehrfach Oriens Christianus. Seine Habilitationsschrift behandelte „Eine jüdisch-hellenistische Rede über den Propheten Jona in armenischer Überlieferung"; in

58 Quelle: Hebrew University [Anm. 57], S. 18. Der erste Band der groß angelegten Reihe, in der diese Sammlung antiker Zeugnisse erscheinen sollte, war 1939 bereits erschienen (von Samuel Klein).

59 Hans Lewy, geb. 1901 in Berlin, gest. am 22. Juli 1945 in Jerusalem. Von 1926 bis 1927 war er Assistent von Hugo Gressmann am Institutum Iudaicum der Universität Berlin (Hugo Gressmann, geb. 1877 verstarb im April 1927 in Chicago während einer Vortragsreise).

60 Die Arbeit erschien in der von Hans Lietzmann, einem Kollegen von Norden, herausgegebenen Reihe Beihefte zur Zeitschrift für die neutestamentliche Wissenschaft und die Kunde der älteren Kirche, Beiheft 9, Gießen, 1929. Die Dissertation wurde im Juli 1926 der Fakultät vorgelegt. - Ein Schwerpunkt der Arbeit liegt auf Philo Alexandrinus, mit dem sich Norden schon als adulescens und wiederum 1921 beschäftigt hat (Norden an Wilamowitz, 8.3.1921); vgl. ABEL, „Studium Berolinense, 2“ [Anm. 3], S. 458 f.

61 Hans Lewy, Lebenslauf, o. J. (nach November 1933), Nachlaß Lewy, Jerusalem. Die Kenntnis des Textes verdanken wir A. Baumgarten.

62 Der Name fehlt in der Liste im Anhang zu Nordens Kleinen Schriften [Anm. 2], S. 689-690. 
seinem Probevortrag am 10. Juli 1933 sprach er über „Das Geschichtswerk des Moses von Chorene".

Das Verfahren gerät in die Abgründe der nationalsozialistischen Universitätspolitik. Die Philosophische Faklutät ${ }^{63}$ stellt in ihrer Sitzung vom 10. Juli 1933 fest, daß Lewys Habilitationsschrift, sein Probevortrag und das Colloquium den Anforderungen „in hervorragendem Maße“ entsprechen. Die venia legendi jedoch wird mit Verweis auf das Gesetz zur Wiederherstellung des Berufsbeamtentums nicht erteilt ${ }^{64}$.

Am 14. Juli 1933, also vier Tage nach dieser Fakultätssitzung, schreibt Norden ein „Gutachten“ für seinen Schüler, das durchaus als Protest gegen diese Entscheidung $\mathrm{zu}$ verstehen ist ${ }^{65}$. In dieser auf genauer Kenntnis beruhenden Würdigung Nordens heißt Lewy „mein persönlicher Schüler“.

Noch in Deutschland hatte Lewy begonnen, die Sammlung griechischer und römischer Texte über Judentum und Juden zu bearbeiten, die Theodor Reinach 1895 zusammengestellt hatte ${ }^{66}$. Das von Lewy bearbeitete Exemplar der Reinachschen Sammlung und weitere Vorarbeiten von Lewy wurden die Grundlage für das moderne Standardwerk Greek and Latin Authors on Jews and Judaism, das Menahem Stern, ein Schüler von Hans Lewy, veröffentlicht hat ${ }^{67}$. Lewys Arbeit über die Juden in der griechischen und römischen Literatur wurde von der „Notgemeinschaft der deutschen Wissenschaft" von 1926-1929 gefördert. Als „Berater“ führt Lewy in seinem zweiten Bericht an die Notgemeinschaft für die Periode 1927/1928 Hans Lietzmann, Eduard Norden und Eduard Meyer von der Berliner Universität sowie Ellbogen, Guttmann und Albeck von der Hochschule für die Wissenschaft des Judentums an ${ }^{68}$. Der Bericht macht deutlich, daß Lewys „Urkundensammlung“ ein Projekt der altertumswissenschaftlichen "Großwissenschaft“ ist, wie sie Theodor Mommsen und Adolf Harnack in Berlin aufgebaut hatten ${ }^{69}$. Ende 1933 bezeichnet Lewy seine „Urkundensammlung

63 Anwesend: Dekan Hartung; Schaeder, Meissner, Sethe, Deubner, Neber, Schwyzer, Lüders, Schulze, Norden.

64 Humboldt-Universität, Akten der Philosophischen Fakultät 3925. Wir danken A. Baumgarten, der uns die Einsicht in dieses Dokument vermittelt hat.

65 Norden, Gutachten, Berlin-Lichterfelde W, Karlstraße 26, den 14. Juli 1933 (Nachlaß Lewy, Jerusalem, 2 S.); ein Adressat ist nicht genannt. Die Kenntnis des Textes verdanken wir A. Baumgarten.

66 Th. ReINACH (1860-1928), Textes d'auteurs grecs et romains, relatifs au Judaïsme, Paris, 1895; Ndr. Hildesheim 1963. - Es ist bemerkenswert, daß auch Schwabe in seinem gleichzeitigen Memorandum eine Überholung, Erneuerung, Vergrößerung der Sammlungen von Reinach plant, s. o. \$2.1.

67 Menahem Stern (1925 Bialystok, Polen; Studium an der HUJ; gest. 1989 in Jerusalem), Greek and Latin Authors on Jews and Judaism, 3 vols., Jerusalem, 1974, 1980, 1984.

68 Lewy, 2. Zwischenbericht 1928, Typoskript, 6 Seiten, Nachlaß Lewy, Jerusalem. Die Kenntnis des Textes verdanken wir A. Baumgarten.

69 Struktur und Projekte dieses Wissenschaftsbetriebs werden aus der distanzierten Perspektive von Franz Overbeck in Basel sehr deutlich, s. OverbecK, Werke und Nachlaß 
mit Kommentar über ,Die Juden in der griechischen und römischen Literatur "“ als

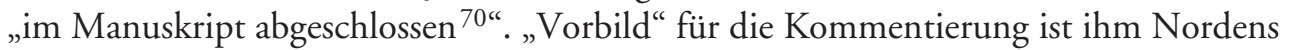
Monographie über Tacitus' Germania ${ }^{71}$.

Im Jahre 1936 wurde Lewy zum Lecturer ernannt; wieder war Norden mit einem Gutachten behilflich. Obschon er selbst sich in dieser Zeit bereits in einer äußerst schwierigen Lage befand, hat er seinen Schüler in Jerusalem unterstützt ${ }^{72}$.

Hans Lewy ist unerwartet früh im Alter von 44 Jahren nach kurzer Krankheit in Jerusalem gestorben. Zu seinem Gedenken sprachen der Rektor der Universität, J. L. Magnes, und zwei Berliner: Gershom Scholem und sein Kollege Max Schwabe. Schwabe zeichnet aus persönlicher Kenntnis ein reiches Bild des akademischen Lebens in Berlin, insbesondere über das Wirken von Eduard Norden und Werner Jaeger, den beiden Betreuern von Lewys Dissertation ${ }^{73}$.

Gershom Scholem, in einem gleichzeitigen Brief an Hannah Arendt, nennt Lewy einen Freund und sagt, als Wissenschaftler sei er "sehr gut" gewesen. Das Urteil Scholems, schon damals Experte für jüdische Mystik, und insofern kompetent für sobria ebrietas, wiegt schwer ${ }^{74}$. Sein Nekrolog evoziert ein komplexes Bild des Berliner Geisteslebens: der Nietzschekultus in großbürgerlichen Salons, bei Bohème und Intelligenz; Stefan George und die Georginen, Friedrich Gundolf und Friedrich Wolters, Kurt Hildebrandt und Hermann Friedmann, der Einfluß Georges auf die jungen Zionisten. Dieser vielschichtige Text zeigt die Berliner Konstellationen der Weimarer Zeit, unter denen Hans Lewy - und Scholem selbst - und ihre Wissenschaft sich ausgebildet haben.

Hubert CANCIK - Hildegard CANCIK-Lindemaier

Postdamer Str. 60

D-12205 Berlin

hicali@t-online.de

3 [Anm. 15], mit den Einleitungen von H. Cancik und H. Cancik-Lindemaier, S. 5 f.; $16 ; 110 ; 446-448$.

70 Lewy, Lebenslauf (Nachlaß Jerusalem).

71 Lewy, 2. Zwischenbericht, p. 5.

72 Die beiden Gutachten Nordens befinden sich im Nachlaß Lewy in den Personal Archives der Nationalbibliothek Jerusalem. Die Kenntnis dieses Bestandes verdanken wir Guy Stroumsa.

73 Eine englische Übersetzung des hebräischen Textes verdanken wir Albert Baumgarten.

74 G. Scholem an Hannah Arendt, Jerusalem, 6. August 1945 (Scholem, Briefe I 1914-1947 [Anm. 55], S. 302 mit Anmerkungen auf S. 448): „Hans Lewy, der Philologe, den Sie wohl auch kennen“ (ist plötzlich gestorben); er sei auch „ein Freund“ von Hans Jonas gewesen. - Scholems hebräische Gedenkrede vom 18.11.45 ist noch nicht in Übersetzung zugänglich. Vgl. G. SCHOLem, Dewarim be-go (Explications and Implications), 2. Teil, Jerusalem, 1975 (S. 479-486). 


\section{Anhang 1: „Schüler/innen von Eduard Norden“"}

A Dissertationen „unter Nordens Mitverantwortung“

(Nach W. Weisshaupt, in E. Norden, Kleine Schriften, S. 689-690; die Liste ist nicht vollständig)

Greifswald:

1898 Otto Altenburg;

Breslau

1901 Stephan Gloeckner;

1903 Ludwig Sniehotta;

1905 Theodor Gollnisch; Hermann Widman; Wilhelm Harendza; Konrat Ziegler;

1906 Richard Gottwald; Josef Seidel;

Berlin

1908 Wilhelm Noetzel;

1909 Kurt Köhler;

1910 Johannes Ehlert; Friedrich Stephan;

1911 Friedrich Bitsch; Erich Pertsch; Alfred Tacke; Arthur Rathke;

1912 Wilhelm Theissen; Fritz Müller-Marquardt; Johannes Focken; Wilhelm Eggerding;

1913 Ernst Hamburger; Alphons Kurfess;

1914 Arthur Saeckel; Carl Virck;

1916 Konrad Heubner; Georg Fürstenau;

1918 Johannes Steinthal;

1919 Wilhelm Neumann;

1920 Ernst Marbach;

1922 Johannes Wegner; Meinrad Paeslack;

1923 Margareta Hirsch;

1924 Irmgard Stutz; Gustav John; Wilhelm Radvann;

1925 Wilhelm Rechnitz;

1926 Werner Jaekel; Karl Springer; Annemaria Holborn geb. Bettmann;

1929 Johannes Georg Wetzel; Alfred Rohde; Gerhard Reincke;

1932 Hans Eberhard Wilhelm; Werner Hartke; Eva Frank; Friedrich Schwarz;

1933 Walter Matzkow; Charlotte Müller;

1934 Rudolf Blum;

1935 Karl Holl;

1936 Elisabeth Haecker.

Hinzuzufügen sind:

Berlin 1926: Elias Bickermann (bei Norden und Wilcken); Hans Lewy (bei Norden und Jaeger). 
B Von Marie Norden, Erinnerungen (1942-44) als „Schüler/in“ von E. Norden bezeichnet:

Christa Behrends; Horst Duchi; Eduard Fraenkel; Harald Fuchs; J. Götte („verheiratet mit einer Schülerin"); Dora Hannasky (Studienrätin in Breslau); Werner Hartke (,mit seiner Schülerin Christa Behrends verheiratet"); Dora Hoffmann; Margarete Jacoby (Nachtrag 2); Karl Kindermann; Pasquali; Schottländer; Fr. Schwarz; von Selchow; Ruth von Velsen; Lissy Velthien; Fritz Wehrli; Ziegler u. a.

\section{Anhang 2: Die Eduard-Norden-Stiftung}

A „Spender der Norden-Stiftung“ (Auswahl) ${ }^{75}$

Akademisch-philologischer Verein, z. H. Herrn Stud.-R. Dr. Paul Gohlke, BerlinSteglitz Gymnasium

$\begin{array}{lll}\text { Aly, Wolfgang } & \text { Prof. Dr. } & \text { Freiburg Br. } \\ \text { Altenburg, Otto } & \text { Prof. Dr. } & \text { Stettin } \\ \text { Ammon, Georg } & \text { Ob.Stud-Dir. i. R., Geh. Stud.-R. Dr. Regensburg } \\ \text { Appel, Carl } & \text { Geh. Reg.-R. Prof. Dr. } & \text { Breslau } \\ \text { Atzrott, Hans } & \text { Stud.-R. } & \text { Berlin-Steglitz } \\ \text { Bang, Martin } & \text { Stud.-R. Dr. } & \text { Berlin } \\ \text { Barwick, Karl } & \text { Prof. Dr. } & \text { Jena } \\ \text { Beckmann, Franz } & \text { Priv.-Doz. Dr. } & \text { Münster i. W. } \\ \text { Bernhard, Max } & \text { Dr. } & \text { Berlin Halensee } \\ \text { Bethe, Erich } & \text { Geh. Hofrat Prof. Dr. } & \text { Leipzig } \\ \text { Biltz, Heinrich } & \text { Prof. Dr. } & \text { Breslau } \\ \text { Bitsch, Friedrich } & \text { Stud.-R. Dr. } & \text { Berlin-Karlshorst } \\ \text { Boesch, Georg } & \text { Ob.-Stud.-Dir. Dr. } & \text { Erfurt } \\ \text { Bölte, Felix } & \text { Prof. Dr. } & \text { Frankfurt } \\ \text { Borchling, Conrad } & \text { Prof. Dr. } & \text { Hamburg } \\ \text { Brackmann, Albert } & \text { Prof. Dr. } & \text { Berlin } \\ \text { Brandl, Alois } & \text { Geh. Reg.-R. Prof. Dr. } & \text { Berlin } \\ \text { Breithaupt, Maximilian } & \text { Gymn.-Dir. Dr. } & \text { Tauberbischofsheim } \\ \text { Breysig, Kurt } & \text { Prof. Dr. } & \text { Rehbrücke b. Berlin } \\ \text { Brock, Arthur } & \text { Prof. } & \text { Leningrad } \\ \text { Bucherer, Fritz } & \text { Gymn.-Dir. Dr. } & \text { Heidelberg } \\ & & \end{array}$

75 Die handschriftliche Liste wurde uns zugänglich gemacht durch Peter Norden (Berlin). Welche weiteren Akten der Norden-Stiftung darüber hinaus existieren (Satzung; Aufstellung der geförderten Personen; Projekte), ist uns unbekannt. 
$\begin{array}{llc}\text { Bulle, Heinrich } & \text { Geh. Reg.-R. Prof. Dr. } & \text { Würzburg } \\ \text { Bultmann, Rudolf, } & \text { Prof. D. } & \text { Marburg } \\ --- & & \\ --- & & \\ --- & \text { Prof. Dr. } & \text { Jena }\end{array}$

B Gedrucktes Widmungsschreiben zur Übergabe der Spenderliste

Herrn Geheimrat Prof. D. Dr. Eduard Norden, Berlin.

Hochverehrter Herr Geheimrat!

Wir überreichen Ihnen heute das Verzeichnis der Kollegen,

Schüler und Freunde, die zu Ihrem sechzigsten Geburtstage

öffentlich bekunden wollen, was nicht allein die Altertumswissenschaft Ihrer Arbeit verdankt. In ihrem Namen dürfen wir Sie bitten, über den Betrag von RM 5257,10 zu verfügen. Den Spendern wäre es eine besondere Freude, wenn er verwendet würde, Ihre Züge von Künstlerhand festhalten zu lassen. Ihre Büste sollte das Berliner Institut für Altertumskunde schmücken, dem Sie zusammen mit den Dioskuren, die es schufen, - nun fast ein Menschenalter vorgestanden haben. Wir hoffen, daß dieser Wunsch Ihrer Freunde und Schüler, der aus objektiver Würdigung Ihrer Stellung im Unterricht unserer ersten Universität geboren ist, Sie bestimmt, Ihre Abneigung gegen persönliche Ehrungen zu überwinden. Andernfalls mögen Sie selbst bestimmen, welchem Zweck der in Dankbarkeit und Freundschaft fir Sie gestiftete Betrag dienen soll.

Der Arbeitsausschuß:

A. Giesecke-Teubner F. Jacoby W. Jaeger

Die ${ }^{76}$ in dem Schreiben genannte Summe ist von einem Spender, der ungenannt bleiben will, auf RM 10000,erhöht worden.

76 Nachtrag auf dem linken unteren Rand. 